\title{
Adaptação de mães à prematuridade: revisão integrativa à luz de Roy
}

\author{
Adaptation of mothers to prematurity: an integrative review in the Roy's vision
}

\author{
Adaptación de las madres a la prematuridad: una revisión integradora a la luz de Roy
}

Paola Panceri dos Reis ${ }^{1 *}$, Eliana Coutinho Evers ${ }^{1}$, Juliana Ollé Mendes ${ }^{1}$, Débora Maria Vargas Makuch ${ }^{1}$.

\section{RESUMO}

Objetivo: Apreender na literatura como se dá o processo de adaptação de mães diante da prematuridade do filho à luz dos modos adaptativos de Roy. Métodos: Trata-se de uma revisão integrativa, utilizando a Biblioteca Virtual em Saúde (BVS) e PubMed® como meios de busca dos estudos, tendo como resultando uma amostra de 8 artigos que atenderam aos critérios de inclusão e exclusão de pesquisa. Para análise dos estudos selecionados foi utilizada a Teoria de Roy como referencial teórico. Resultados: Foi possível identificar que a prematuridade tem impacto em todos os quatro modos adaptativos propostos pela Teoria de Roy, que explica a adaptação da pessoa diante de um evento/estímulo que modifica sua vida, sendo maior o impacto nos modos autoconceito e interdependência. Além disso, é possível perceber uma forte ligação entre esses modos, que também servem de mecanismos adaptativos. Considerações finais: Percebe-se que a prematuridade impacta diretamente os quatro modos adaptativos propostos pela Teoria da adaptação de Callista Roy, tendo maior impacto no modo autoconceito, seguido de função de papel e interdependência e, por último, modo fisiológico. Evidencia-se o grande papel do enfermeiro como ferramenta para uma adaptação mais efetiva.

Palavras-chave: Recém-nascido pré-termo, Relações mãe-filho, Adaptação.

\section{ABSTRACT}

Objective: To apprehend in the literature how mothers adapt to the prematurity of their children in the light of Roy's adaptive ways. Methods: This is an integrative review, using the Virtual Health Library and PubMed® as means of searching for studies, resulting in a sample of 8 articles that met the inclusion and exclusion criteria of the research. To analyze the selected studies, Roy's theory was used as a theoretical framework. Results: It was possible to identify that prematurity has an impact on all four adaptive modes proposed by Roy's Theory, which explains the person's adaptation to an event / stimulus that changes their life, with a greater impact on the self-concept and interdependence modes. In addition, it is possible to perceive a strong link between these modes, which also serve as adaptive mechanisms. Final Considerations: It is noticed that prematurity directly impacts the four adaptive modes proposed by Callista Roy's Theory of Adaptation, having a greater impact on the self-concept mode, followed by paper function and interdependence and, finally, physiological mode. The great role of nurses is evidenced as a tool for a more effective adaptation.

Keywords: Infant premature, Mother-child relations, Adaptation.

\section{RESUMEN}

Objetivo: Conhecer na literatura como as mães se adaptam à prematuridade de seus filhos à luz das formas adaptativas de Roy. Métodos: Se trata de una revisión integradora, utilizando la Biblioteca Virtual en Salud y PubMed® como medio de búsqueda de estudios, resultando una muestra de 8 artículos que cumplieron con los criterios de inclusión y exclusión de la investigación. Para analizar los estudios seleccionados, se utilizó la teoría de Roy como marco teórico. Resultados: Se pudo identificar que la prematuridad tiene un impacto en los cuatro modos adaptativos propuestos por la Teoría de Roy, que explica la adaptación de la persona a un evento / estímulo que cambia su vida, con un mayor impacto en los modos de autoconcepto e interdependencia. Además, es posible percibir un fuerte vínculo entre estos modos, que también sirven como mecanismos adaptativos. Consideraciones finales: Se observa que la prematuridad impacta directamente en los cuatro modos adaptativos propuestos por la Teoría de la Adaptación de Callista Roy, teniendo un mayor impacto en el modo de autoconcepto, seguido de la función de papel e interdependencia y, finalmente, el modo fisiológico. Se evidencia el gran papel de las enfermeras como herramienta para una adaptación más efectiva.

Palabras clave: Recién nacido prematuro, Relaciones madre-hijo, Adaptación.

${ }^{1}$ Faculdades Pequeno Príncipe, Curitiba - PR. *E-mail: paapreis@gmail.com 


\section{INTRODUÇÃO}

A prematuridade é definida pelo nascimento de um bebê antes de completar 37 semanas de gestação. No Brasil, aproximadamente $10 \%$ dos recém-nascidos vivos são prematuros (BRASIL, 2017). O bebê prematuro, devido a sua fragilidade, apresenta risco maior de desenvolver diversas complicações após o nascimento como: hemorragias cerebrais, infecções, paralisia cerebral, atraso no desenvolvimento neuropsicomotor, problemas respiratórios, problemas cognitivos, entre outros (TAMEZ R, 2013). Cerca de $65 \%$ das mortes nos primeiros 28 dias de vida são de recém-nascidos prematuros e a prematuridade é a responsável pela maioria das internações em Unidades de Terapia Intensiva Neonatais (UTINEO) (ONU, 2018).

Uma gestação tem duração média de 280 dias, cerca de 40 semanas. Há um preparo da mulher para o tornar-se mãe durante a gravidez. No decorrer desse tempo, a gestante passa por mudanças biológicas, psicológicas e sociais, e a maneira como ela lida com esse período de fortes mudanças influencia diretamente na formação da maternidade e na relação mãe-filho. O parto é o momento de encontro e reconhecimento entre o bebê a mãe. Quando esse momento ocorre de forma prematura seu significado é rompido e, o processo torna-se doloroso e traumático, carregado de angústia, medo, aflição, de enfrentamentos inesperados e dificuldades do início do trabalho de parto até o nascimento em si (FERRARI AG e DONELLI TMS, 2010).

O puerpério da nova mãe inicia-se acompanhado das dificuldades de uma nova rotina hospitalar com cuidados intensivos, do medo da morte de seu filho e da impossibilidade de acalentar e cuidar de seu bebê imediatamente. Esse evento demanda diversas mudanças e complicações, e cria um ambiente de incertezas e medo à puérpera que ainda está no processo de transição ao mundo materno, sendo necessária sua adaptação a essa nova realidade (ZANFOLIM LC, et al., 2018).

Diante disso e de outros eventos semelhantes, a enfermeira Callista Roy criou a Teoria da Adaptação, que traz os modos adaptativos enfrentados por pessoas diante de um evento (estímulo) como esse. A construção de teorias de enfermagem visa organizar o cuidado de forma que englobe o cliente holisticamente, ou seja, sua dimensão fisiológica, espiritual, emocional e social. Além disso, promove a criação de cuidado mais crítico, científico e reflexivo e um olhar diferenciado à execução da assistência à saúde. (COELHO SMS e MENDES IMD, 2011).

Inicialmente, Roy definiu seus metaparadigmas, a pessoa, ambiente, saúde e enfermagem. Pessoa é um sistema holístico e adaptativo, que não é necessariamente um indivíduo, pode ser também uma família, grupo, comunidade ou sociedade. Além disso, a pessoa é um sistema adaptativo com unidades relacionadas a elas, como input (estímulos), nível de adaptação, processos de controle, output (respostas) e processos de feedback ou retroalimentação. A autora propõe quatro modos adaptativos, sendo eles: modo fisiológico, autoconceito, função de papel e interdependência. (ANDREWS e ROY apud IVO ML, 2007).

Diante das mudanças que o nascimento prematuro traz e da adaptação que é necessária às mães emerge a questão norteadora: $O$ que versa a literatura acerca da adaptação de mães à prematuridade do recémnascido?

Para tanto, o objetivo deste estudo é: Apreender na literatura como se dá o processo de adaptação de mães diante da prematuridade do filho à luz dos modos adaptativos de Roy.

\section{MÉTODOS}

Para a construção desse estudo foi utilizado o método de revisão integrativa fundamentado nas etapas propostas por Mendes KDS, et al., (2019). Articulado a este método optou-se pela abordagem qualitativa, alicerçada pelos Modos Adaptativos de Roy.

Para a busca e seleção dos estudos foram escolhidos os bancos de dados BVS e PubMed®, e utilizados os seguintes descritores "Adaptação and relações mãe-filho and recém-nascido pré-termo" e "Adaptation and mother-child relations and preterm newborn". Adotados como critérios de inclusão: artigos disponíveis, idioma (inglês, português e espanhol), período de 2010 a maio de 2020 e estudos relevantes ao tema. Os critérios de exclusão utilizados foram: artigos não disponíveis, teses e dissertações, período anterior a 2010 e posterior a maio de 2020, artigos redundantes e não relevantes ao tema. 
Para a efetivação desta pesquisa, foram selecionados 8 artigos que atendiam os critérios de inclusão estabelecidos para este estudo, demonstrado abaixo na Figura 1.

Figura 1 - Fluxograma de seleção dos estudos.

Bases de dados: BVS e PubMed

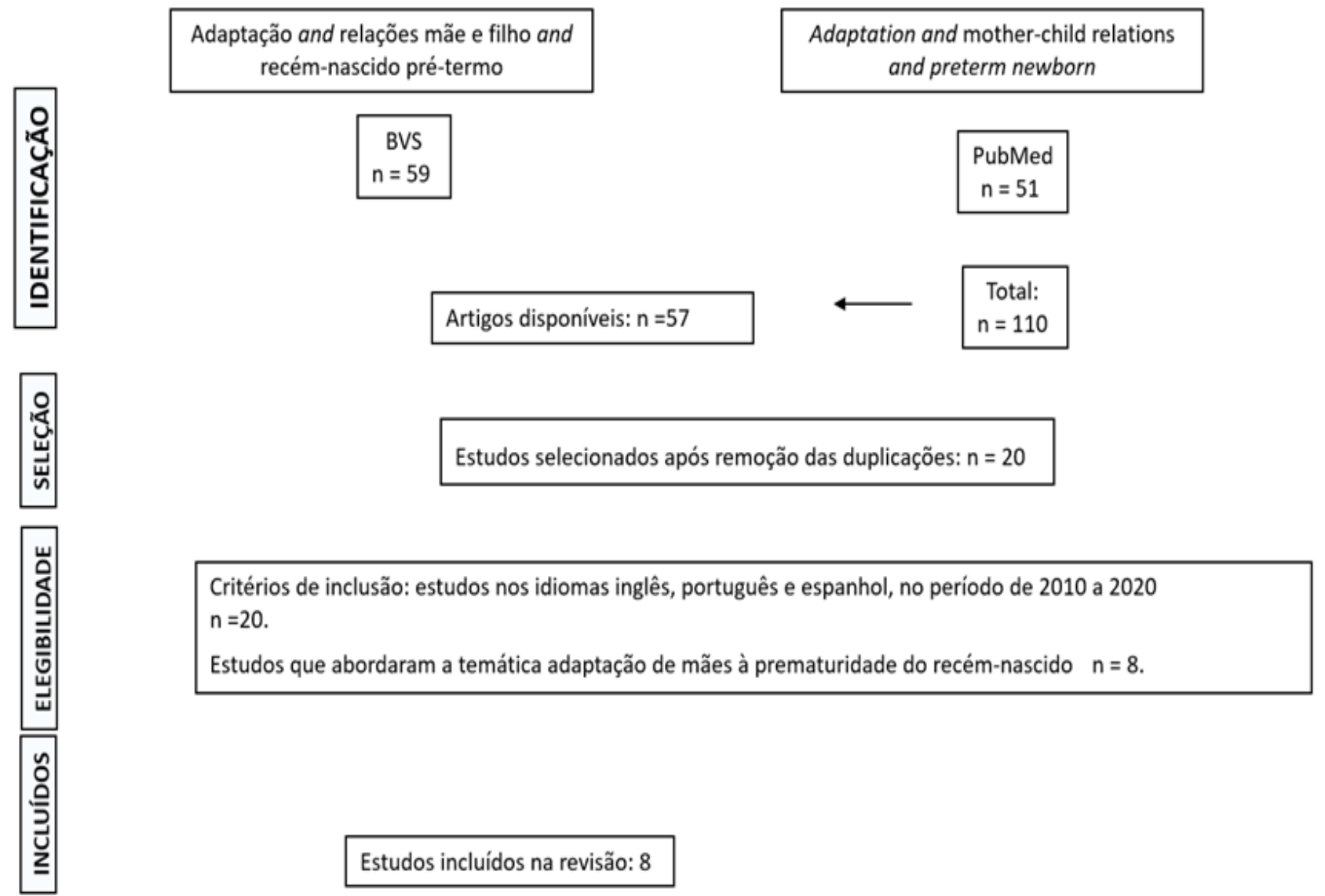

Fonte: Reis PP, et al., 2020.

\section{RESULTADOS}

Depois de selecionar os dados extraídos dos estudos primários é necessário avaliar de forma crítica cada variável (MENDES KDS, et al., 2019). As variáveis que se mostraram mais relevantes nesse estudo foram: ano, tipo de abordagem dos estudos, local de estudo e modos adaptativos citados. Referindo-se ao ano de publicação, $75 \%$ foram publicados nos últimos 5 anos. A abordagem qualitativa prevalece $(87,5 \%)$, visto que o tema leva em conta a experiência pessoal e a subjetividade de cada participante.

Em relação ao local de realização dos estudos, $62,5 \%$ foram realizados em UTINEO, onde se encontram mães de recém-nascidos prematuros que estão passando por um enfrentamento e adaptação maior do que aquelas mães que já receberam alta.

Quanto aos modos adaptativos de Roy, o modo autoconceito foi citado em 7 (87,5\%) artigos, seguido de função de papel e interdependências, ambos com citação em 4 (50\%) e por último o modo fisiológico citado em $2(25 \%)$ artigos, os quais serão apresentados a seguir.

A fim de organizar as principais informações de cada estudo selecionado foi criado um quadro com os seguintes tópicos: ano de publicação, autores, título do estudo, tipo de estudo, local de estudo e modos adaptativos citados (Quadro 1). 


\section{Revista Eletrônica Acervo Saúde / Electronic Journal Collection Health | ISSN 2178-2091}

Quadro 1 - Organização dos estudos quanto ao ano de publicação, autor(es), título do artigo, tipo de estudo, local de estudo e modo adaptativo.

\begin{tabular}{|c|c|c|c|c|c|}
\hline Ano & Autor(es) & Título do artigo & Tipo do estudo & Local de estudo & Modo adaptativo \\
\hline 2019 & $\begin{array}{l}\text { Nazareth IV, } \\
\text { et al. }\end{array}$ & $\begin{array}{l}\text { Riscos gestacionais e o nascimento } \\
\text { prematuro: enfrentamento para a } \\
\text { maternagem }\end{array}$ & $\begin{array}{l}\text { Estudo descritivo, com } \\
\text { abordagem qualitativo, } \\
\text { baseado na etnoenfermagem }\end{array}$ & $\begin{array}{l}\text { Ambulatório de follow-up de } \\
\text { um hospital universitário do } \\
\text { Rio de Janeiro }\end{array}$ & $\begin{array}{l}\text { Autoconceito, Função } \\
\text { de papel } \quad \text { e } \\
\text { Interdependência }\end{array}$ \\
\hline 2012 & Lee $S$, et al. & $\begin{array}{l}\text { Does Activity Matter: an Exploratory Study } \\
\text { among Mothers with Preterm Infants }\end{array}$ & $\begin{array}{l}\text { Exploratório descritivo com } \\
\text { abordagem quantitativa }\end{array}$ & $\begin{array}{l}3 \text { UTINEO de uma cidade do } \\
\text { sul dos EUA }\end{array}$ & Função Fisiológica \\
\hline 2014 & $\begin{array}{l}\text { MELO ECJ, } \\
\text { et al. }\end{array}$ & $\begin{array}{l}\text { A voz da mulher-mãe de prematuro na } \\
\text { unidade neonatal: uma abordagem } \\
\text { fenomenológica. }\end{array}$ & $\begin{array}{l}\text { Estudo qualitativo, } \\
\text { fenomenológico, } \\
\text { fundamentado no pensamento } \\
\text { de Martin Heidegger }\end{array}$ & $\begin{array}{l}\text { UTINEO de um hospital } \\
\text { universitário da rede pública } \\
\text { da cidade do Rio de Janeiro }\end{array}$ & Autoconceito \\
\hline 2017 & $\begin{array}{l}\text { ROSSMAN } \\
\text { B, et al. }\end{array}$ & $\begin{array}{l}\text { Resilience in Mothers of Very-Low-Birth- } \\
\text { Weight Infants Hospitalized in the NICU. }\end{array}$ & $\begin{array}{l}\text { Estudo narrativo com } \\
\text { abordagem qualitativa }\end{array}$ & UTINEO de Chicago & $\begin{array}{l}\text { Autoconceito, Função } \\
\text { de Papel } \quad \text { e } \\
\text { Interdependência }\end{array}$ \\
\hline 2011 & $\begin{array}{l}\text { Brandon } \\
\mathrm{DH} \text {, et al. }\end{array}$ & $\begin{array}{l}\text { Emotional Response of Mothers of Late- } \\
\text { Preterm and Term Infants }\end{array}$ & $\begin{array}{l}\text { Estudo descritivo abordagem } \\
\text { qualitativa }\end{array}$ & $\begin{array}{l}\text { Uma unidade de pós parto de } \\
\text { um centro médico acadêmico. }\end{array}$ & $\begin{array}{l}\text { Autoconceito e função } \\
\text { de papel }\end{array}$ \\
\hline 2017 & $\begin{array}{l}\text { VERONEZ } \\
\text { M, et al. }\end{array}$ & $\begin{array}{l}\text { Experience of mothers of premature } \\
\text { babies from birth to discharge: notes of } \\
\text { field journals. }\end{array}$ & $\begin{array}{l}\text { Estudo exploratório descritivo } \\
\text { com abordagem qualitativa }\end{array}$ & Hospital do sul do Brasil & $\begin{array}{l}\text { Autoconceito } \\
\text { interdependência }\end{array}$ \\
\hline 2016 & $\begin{array}{l}\text { Henderson } \\
\mathrm{J} \text {, et al. }\end{array}$ & $\begin{array}{l}\text { Impact of preterm birth on maternal well- } \\
\text { being and women's perceptions of their } \\
\text { baby: a population-based survey }\end{array}$ & $\begin{array}{l}\text { Estudo exploratório descritivo } \\
\text { com abordagem qualitativa }\end{array}$ & Inglaterra (não especificado) & $\begin{array}{l}\text { Função fisiológica e } \\
\text { autoconceito }\end{array}$ \\
\hline 2016 & $\begin{array}{l}\text { NCUBE RK, } \\
\quad \text { et al. }\end{array}$ & $\begin{array}{l}\text { A life uncertain - My baby's vulnerability: } \\
\text { Mothers' lived experience of connection } \\
\text { with their preterm infants in a Botswana } \\
\text { neonatal intensive care unit }\end{array}$ & $\begin{array}{l}\text { Estudo fenomenológico, } \\
\text { exploratório e descritivo com } \\
\text { abordagem qualitativa }\end{array}$ & $\begin{array}{l}\text { unidade infantil de cuidados } \\
\text { intensivos / UTINEO de um } \\
\text { hospital de referência em } \\
\text { Gaborone, Botsuana }\end{array}$ & $\begin{array}{l}\text { Autoconceito, função de } \\
\text { papel e } \\
\text { interdependência. }\end{array}$ \\
\hline
\end{tabular}

Fonte: REIS PP, et al., 2020.

REAS/EJCH | Vol.13(1) | e5827 | DOI: https://doi.org/10.25248/reas.e5827.2021 Página 4 de 8 


\section{DISCUSSÃO}

O nascimento prematuro é um evento que acarreta estresse sobre a família que vivencia essa situação, principalmente as mães dos recém-nascidos (HENDERSON J, et al., 2016). A transição de mulher para mãe começa com recém-nascido distante da mãe, e é caracterizada por confusão, tristeza, medo, choque e incerteza sobre a saúde do bebê. Os sonhos e idealizações das mães de aninhar, conhecer e sentir seus filhos foram substituídos pelo trauma das primeiras visitas à UTINEO. Isso gera impactos fisiológicos, psicológicos e sociais, sendo necessária a adaptação a situação (ROSSMAN B, et al., 2017).

\section{Teoria da adaptação}

A construção de teorias de enfermagem visa organizar o cuidado de forma que englobe o cliente holisticamente, ou seja, sua dimensão fisiológica, espiritual, emocional e social. Além disso, promove a criação de cuidado mais crítico, científico e reflexivo e um olhar diferenciado à execução da assistência à saúde. Callista Roy propõe quatro modos adaptativos, sendo eles: modo fisiológico, autoconceito, função de papel e interdependência (COELHO SMS e MENDES IMD, 2011).

Inicialmente, Roy definiu seus metaparadigmas, a pessoa, ambiente, saúde e enfermagem. Pessoa é um sistema holístico e adaptativo, que não é necessariamente um indivíduo, pode ser também uma família, grupo, comunidade ou sociedade. Além disso, a pessoa é um sistema adaptativo com unidades relacionadas a elas, como input (estímulos), nível de adaptação, processos de controle, output (respostas) e processos de feedback ou retroalimentação (SALDANHA EA, et al., 2012).

$O$ ambiente é entendido como todas as influências que afetam e modificam a vida das pessoas. A definição de saúde é oriunda dos conceitos de pessoa e ambiente, ela é reflexo da adaptação, é o processo de ser e se tornar uma pessoa íntegra, atingindo as metas de sobrevivência, crescimento, reprodução e domínio. (ANDREWS e ROY apud IVO, 2007).

As respostas adaptativas são manifestadas através dos modos adaptativos, sendo esses as vias para o processo adaptativo. Os quatro modos adaptativos descritos na Teoria da Roy são: Função Fisiológica, Auto Conceito, Função do Papel e Interdependência (FREITAS MC, et al., 2014).

O modo Função fisiológica diz respeito as respostas referentes as cinco atividades fisiológicas básicas, sendo elas: oxigenação, eliminação, nutrição, proteção, atividade/repouso. Além das cinco atividades fisiológicas possui quatro processos de regulação: sentidos, líquidos e eletrólitos, função endócrina e função neurológica. Nesse modo é feita a avaliação da integridade fisiológica da pessoa (SALDANHA EA, et al., 2012).

O Auto Conceito é um modo bem amplo com várias subdivisões, aonde se avalia a integridade psíquica da pessoa. Leva em consideração os aspectos psicológicos, morais e espirituais da pessoa, suas crenças, valores e sentimentos sobre si mesmo, formados em uma determinada situação, com percepções internas e de terceiros. Trata-se de como a pessoa se percebe, da questão: quem sou eu? (MEDEIROS et al., 2015).

O modo Função de Papel corresponde aos papéis sociais que a pessoa desempenha na sociedade, aonde avalia-se a integridade social do indivíduo. Os papéis sociais desempenhados pela pessoa são parte dela e de sua vida, seu comportamento, relacionamento e expectativas em relação a ela mesmo e aos outros pode afetar a sua integridade social (DIAZ LJR e CRUZ DALM, 2017).

Por fim, o modo da Interdependência relaciona-se as interações sociais, manutenção de relacionamentos, sobre o afeto, o dar e receber carinho, atenção, respeito e valor. Nesse modo é avaliado a integridade afetiva (SALDANHA EA, et al., 2012).

\section{Modo fisiológico}

De acordo com Lee S, et al. (2012), o sono é fundamental para a vida dos seres humanos, tem relação direta com a qualidade de vida, sendo um dos cinco componentes das atividades fisiológicas básicas. A maternidade já tende a alterar a rotina de atividade e repouso quando ocorre em condições habituais e esse fator não é diferente para as mães de prematuros, mesmo aquelas cujos bebês recebem os cuidados diretos de profissionais de saúde. 
Mães de prematuros têm uma maior probabilidade de problemas e alterações no sono, fadiga e ritmos de atividade/repouso em comparação com mães de recém-nascidos a termo. Reforçando esse dado, segundo Henderson J, et al. (2016), mães de recém-nascidos prematuros apresentam mais fadiga e cansaço no primeiro mês pós-parto e esse comportamento se repete em mães de recém-nascidos prematuros extremos 3 meses após o parto. Este fator impacta negativamente na sua qualidade de vida, visto que o cansaço e falta de repouso aumentam os níveis de estresse materno, eleva as chances do surgimento de depressão pósparto, abala o relacionamento mãe e filho e, com isso, afeta também outros modos adaptativos.

\section{Autoconceito}

Apesar de cada nascimento prematuro ter sua singularidade para cada mãe que o vivencia, os sentimentos de medo, insegurança e fragilidade são comuns em vários relatos. A angústia, a ansiedade e medo tornamse presentes na transição do papel de gestante a mãe em meio a um cenário de UTINEO e risco eminente de morte do bebê. Além disso, a insegurança em relação a ser capaz de cuidar de um bebê que nasceu antes do previsto também é prevalente. Em muitos casos, há também o sentimento de culpa por não sustentar a situação (NAZARETH IV, et al., 2019).

Estar num ambiente diferente do habitual, cheio de equipamentos e profissionais da saúde também gera grande desconforto e sentimentos negativos. A incompreensão do que está acontecendo e dos termos técnicos da rotina hospitalar gera frustração e, muitas vezes, apreensão de "atrapalhar" o serviço dos profissionais, o que pode acarretar no afastamento da rotina de cuidados do bebê, tão importante para a criação do vínculo mãe e filho e para o processo de tornar-se mãe (MELO RCJ, et al., 2014).

Estar dentro da rotina hospitalar pode afastar a mãe de seu filho, fator esse que um sentimento de culpa materna por não se sentir conectada ao seu filho. A hospitalização dificulta o contato da mãe diretamente com o filho que é essencial para a criação de vínculo, para o reconhecimento daquele bebê como seu e, consequentemente, para a apropriação do papel materno. Esse fator gera frustração, culpa e tristeza e acaba por afetar diretamente na transição para o papel materno (BRANDON DH, et al., 2011). Dados colhidos no estudo de Henderson J, et al. (2016) mostram uma diferença significativa de mães de recém-nascidos prematuros que não sentem que o seu filho lhe pertence $(5 \%)$ em comparação a mães de recém-nascidos a termo $(0,6 \%)$.

Além disso, há relatos de uma profunda tristeza e frustração por não poderem realizar primeiros cuidados com o seu bebê. Em recém-nascidos prematuros hospitalizados, geralmente, os cuidados de higiene, alimentação e outros são realizados pelos profissionais de enfermagem das unidades de internação, além daqueles que são privativos dos profissionais, e, dificilmente, as mães conseguem participar devido a rotina do setor e até mesmo pela situação de saúde do bebê (VERONEZ M, et al., 2017). O contato pele a pele é extremamente benéfico. Só o simples gesto de segurar seu filho no colo confere às mães uma sensação de confiança, a qual substitui o medo, frustração e melhora seu bem-estar (NCUBE RK, et al., 2016).

Como mecanismo de adaptação, muitas mães criam resiliência, encontrando força em seus filhos. Resiliência é a capacidade de enfrentar eventos adversos da vida, um processo adaptativo positivo, que geralmente resulta em uma adaptação plena da situação. Esse processo não é imediato, acontece em etapas, primeiro é experimentar o trauma e segundo entendê-lo e desenvolver a resiliência. Chorar, orar, pedir apoio, buscar informações, focar em seu filho são alguns dos mecanismos utilizados para criar a resiliência e adaptar-se efetivamente (ROSSMAN B, et al., 2017).

\section{Função de papel}

Os papéis que a mulher desempenha e aqueles que ela começará a desempenhar após o parto são afetados com a prematuridade. A aquisição do papel materno ocorre tardiamente, visto que as puérperas se sentem despreparadas com a situação e apresentam dificuldades de conexão com seus bebês (BRANDON $\mathrm{DH}$, et al., 2011).

O papel "mãe" não é adquirido do dia para a noite. Ele ocorre com a vivência da maternagem em si, com a experiência do contato com seu filho. Quando se fala em mães de recém-nascidos prematuros, assumir esse papel é diferente do esperado. A transição do papel de mulher para o de mãe ocorreu num ambiente repleto de confusão e incertezas, totalmente diferente do idealizado (ROSSMAN B, et al., 2017). 
A limitação do contato e interação da mãe com seu bebê, em decorrência da internação da criança, acarreta dificuldade na criação de vínculo e, além disso, é responsável pelo sentimento de perda do papel materno referido por diversas mães (LEE S, et al., 2012).

Em tese, a mãe é a responsável pelo seu filho, contudo para mães com bebês hospitalizados há um grande desejo de poder assumir a responsabilidade do cuidado ao seu filho na prática e não ser apenas uma "espectadora" (NCUBE RK, et al., 2016). Várias ações fazem com que a mulher se sinta participante ativa da vida do seu filho, o cuidado direto a ele é uma delas. Quando é oportunizado à mulher participar e realizar os cuidados ao seu bebê, ela inicia uma conexão real e tangível, facilitadora na relação mãe-filho e na obtenção do papel de mãe. O estudo relata que, conforme a conexão mãe e filho aumentava, o amor sentido pela mãe servia como fonte de forças para o enfrentamento da situação, facilitando a adaptação (NAZARETH IV, et al., 2019).

A alta hospitalar é um dos momentos mais esperados pelas mães. Esse momento significa a quebra da rotina hospitalar e o início dos cuidados da mãe para com o bebê, onde ela assume de forma plena o papel de cuidadora de seu filho. Mesmo sendo tão esperado, essa nova fase necessita de adaptação, pois será uma nova rotina, uma nova experiência. Nesse contexto, o profissional enfermeiro é essencial, pois age como educador, orienta a forma adequada de realizar os cuidados, elimina dúvidas e faz com que essa mãe se sinta mais segura (NAZARETH IV, et al., 2019).

\section{Interdependência}

As relações interpessoais são de extrema importância para o ser humano. Em uma situação inesperada, podem ajudar ou até mesmo piorar a situação, sendo uma arma facilitadora ou dificultadora do processo adaptativo. Percebe-se que a relação com os profissionais de saúde neste contexto tem grande impacto na adaptação das mães à prematuridade, tanto positiva quanto negativamente. $O$ enfermeiro é o profissional que mais tem contato com a mulher durante o internamento do prematuro, sendo responsável por boa parte das orientações sobre o estado de saúde da criança, também no auxílio ao primeiro contato mãe e filho, tendo o poder de aliviar a tensão e ansiedade materna durante o processo (NAZARETH IV, et al., 2019).

Interações positivas e a boa comunicação dos profissionais da saúde com as mães, principalmente aqueles do setor da UTINEO garantem benefícios à adaptação materna, as puérperas sentem-se mais acolhidas no setor, mais confortáveis e confiantes em cuidar de seus filhos e deixá-los sob cuidados da equipe. Ouvir e entender as dores e anseios das mães é uma das habilidades que auxiliam os profissionais da saúde na adaptação materna. Sentir-se apoiada e compreendida faz com que a puérpera tenha forças para lidar com a sua realidade (NCUBE RK, et al., 2016). Apesar disso, a interação com os profissionais pode também ser negativa. A rotina fatigante da UTINEO por muitas vezes não permite que o profissional possa dar a atenção individualizada necessária a cada mãe e, com isso, cria-se uma relação conturbada, que gera ansiedade e frustração materna, atrapalhando o processo de adaptação (ROSSMAN B, et al., 2017).

A relação familiar é um grande pilar de sustentação materna. Uma boa rede de apoio advinda de pessoas que participam do convívio diário é essencial para uma adaptação efetiva. Apesar disso, muitas mães relatam se sentirem incompreendidas por amigos próximos e familiares. Tal fato pode ocasionar um distanciamento social da mulher com as pessoas de sua proximidade, fazendo com que ela se sinta sozinha Além do apoio de familiares e amigos, o apoio de mães que passam por situações semelhantes é muito positivo. Dividir experiências, ser ouvida, tirar dúvidas e compartilhar sentimentos fazem com que a puérpera se sinta mais acolhida e confiante para enfrentar a prematuridade de seu filho. A criação de laços com pessoas que passaram pela mesma situação auxilia na compreensão de seus sentimentos e a lidar melhor com eles (ROSSMAN B, et al., 2017).

Por fim, uma das relações mais importantes é a relação mãe e filho. Com o passar dos dias, inicia-se o processo de conhecimento do seu filho e encontro com a maternidade. À medida que esta relação é construída, a mulher se empodera de seu papel materno, o sentimento de amor ao seu filho aumenta junto com sua confiança (VERONEZ M, et al., 2017).

Este estudo teve como limitação o número reduzido de artigos publicados sobre o tema. Sugere-se que a produção científica sobre a temática se amplie, o que certamente motivará mais reflexões, inclusive durante a formação profissional. 


\section{CONSIDERAÇÕES FINAIS}

A prematuridade é um evento que impacta negativamente a mulher em todas as áreas de sua vida, deste modo, afeta os quatro modos adaptativos propostos pela teoria da adaptação de Callista Roy, tendo maior impacto no modo de autoconceito, em segundo lugar nos modos função de papel e interdependência e por último o modo fisiológico. Percebe-se que os modos adaptativos propostos por Roy são interrelacionados, um tendo impacto no outro de forma contínua. A adaptação ocorrerá em algum momento a todas as mães, porém existem mecanismos que facilitam esse processo. Nesse sentido, o enfermeiro atua como importante ferramenta adaptativa. Esse profissional tem dupla função na unidade de terapia intensiva neonatal: cuidar do recém-nascido prematuro e da mãe. É ele quem irá acolher a mulher, realizará educação constante acerca dos cuidados do bebê, tirar dúvidas, compartilhará informações importantes sobre seu estado de saúde e tratamento e, muitas vezes, acalmará e ouvirá seus medos e anseios, deste modo, seu trabalho facilita a adaptação eficaz da mãe à prematuridade.

\section{REFERÊNCIAS}

1. BRANDON DH, et al. Emotional Responses of mother of late-preterm and term infants. Journal of Obstetric Gynecologic \& Neonatal Nursing, 2011, 40 (6): 719-731.

2. BRASIL, 2017. In: Bebês Prematuros. Brasil: Ministério da Saúde. Disponível em: http://www.saude.gov.br/noticias/823-assuntos/saude-para-voce/40775-bebes-prematuros. Acesso em: 25 ago. 2019.

3. COELHO SMS, MENDES IMD. Da pesquisa à prática de enfermagem aplicando o modelo de adaptação de Roy. Escola Anna Nery, 2011, 15(4): 845-850.

4. DIAZ LJR, CRUZ, DALM. Modelo de adaptação em um ensaio clínico controlado com cuidadores familiares de pessoas com doenças crônicas. Texto contexto - enferm. 2017, 26(4): 1-10.

5. FERRARI AG, DONELLI TMS. Tornar-se mãe e prematuridade: considerações sobre a constituição da maternidade no contexto do nascimento de um bebê com muito baixo peso. Contextos Clínicos, 2010, 3 (2): 106-112.

6. FREITAS MC ,et al . Idosos residentes em uma instituição de longa permanência: adaptação à luz de Callista Roy. Rev. bras. enferm. 2014, 67(6): 905-912.

7. HENDERSON J, ei al. Impact of preterm birth on maternal well-being and women's perceptions of their baby: a population-based survey. BMJ Open, 2016, 6(10): 1-8.

8. IVO, ML. Modelo de Adaptação de Roy e sua aplicação através do processo de enfermagem, a portadores de anemia falciforme. Campo Grande: Universidade Federal Mato Grosso do Sul, 2007, 228 p.

9. LEE S, et al. Does activity matter: an exploratory study among mothers with preterm infants?. Arch Womens Ment Health, 2012, 15(3): 185-192.

10. MEDEIROS LP, et al. Modelo de Adaptação de Roy: revisão integrativa dos estudos realizados à luz da teoria. Revista Rene, 2015,16(1): 132-140.

11. MELO RCJ, el al. A voz da mulher-mãe de prematuro na unidade neonatal: uma abordagem fenomenológica. Online Brazilian Journal Nursing, 2014, 13(2): 198-216.

12. MENDES KDS, et al. Uso de gerenciador de referências bibliográficas na seleção dos estudos primários em revisão integrativa. Texto context, 2019, 28: 1-13.

13. NAZARETH IV, et al. Riscos gestacionais e o nascimento prematuro: enfrentamento para a maternagem. Rev. enferm. UFPE on-line, 2019, 13(4): 1030-1039.

14. NCUBE RK, et al. A life uncertain - My baby's vulnerability: Mother's lived experience of connection with their preterm infants in a Botswana neonatal intensive care unit. Curationis, 2016, 39(1):1-9.

15. ONU, 2018. In: OMS: cerca de 30 milhões de bebês nascem prematuros por ano no mundo. Brasil: Organização das Nações Unidas. Disponível em: https://nacoesunidas.org/oms-cerca-de-30-milhoes-de-bebes-nascem-prematurospor-ano-no-mundo/. Acesso em: 25 ago. 2019.

16. ROSSMAN B, et al. Resilience in mothers of very-low-birth-weight infants hospitalized in the NICU. J Obstet Gynecol Neonatal Nurs., 2017, 43(3): 434-445.

17. SALDANHA EA, et al. A teoria de Callista Roy, a NANDA e o cuidado ao paciente prostatectomizado. Revista Enfermagem UERJ, 2012, 20(2): 764-70.

18. TAMEZ R. Enfermagem na UTI Neonatal. 5.ed. Rio de Janeiro: Guanarabara; 2013; 386p.

19. VERONEZ M, et al. Experience of mothers of premature babies from birth to discharge: notes of field journals. Rev. Gaúcha Enfermagem, 2017, 38(2):1-8.

20. ZANFOLIM LC, et al. Dificuldades Vivenciadas pelas Mães na Hospitalização de seus Bebês em Unidades Neonatais. Psicologia: Ciência e Profissão, 2018, 38(1): 22-35. 